Note: This is a pre-copy-editing, author-produced PDF of an article accepted for publication in Substance Use \& Misuse following peer review. The definitive publisher-authenticated version [McElrath K (2005) MDMA and sexual behavior: ecstasy users' perceptions about sexuality and sexual risk, Substance Use \& Misuse, 40:9, 1461-1477] is available online at

http://www.informaworld.com/smpp/title $\sim \mathrm{db}=$ all content $=\mathrm{g} 714012467$

\title{
MDMA and Sexual Behavior: Ecstasy Users' Perceptions About Sexuality and Sexual Risk
}

\author{
KAREN MCELRATH \\ School of Sociology and Social Policy, Belfast, Ireland \\ Published in Substance Use \& Misuse,(2005) 40:9,1461—1477
}

This study examines the relationship between MDMA (Ecstasy), sexual behavior, and sexual risk taking. The sample consisted of 98 current and former users of MDMA. Several strategies were utilized to recruit respondents and data were collected through in-depth interviews during 1997 and 1998. The majority of respondents had used MDMA during the 6-month period prior to the interview and a large percentage had consumed the drug on 100 occasions or more. Most respondents reported feelings of emotional closeness while consuming MDMA but without the desire for penetrative sex. Others, however, reported that MDMA increased sexual arousal and some respondents (in particular gay and bisexual females) had used MDMA specifically for sexual enhancement. Sexual risk taking (e.g., having multiple partners, engaging in sex without a condom) was prevalent among respondents who did engage in sexual activity during MDMA episodes. Explanations for the findings are offered and implications for prevention/intervention are discussed.

Keywords MDMA; ecstasy; sexual behavior

\section{Introduction}

Although a patent for 3,4-methylenedioxymethamphetamine (MDMA) was issued in 1914 (Shulgin, 1986), "recreational"a use of the drug did not surface until the 1970s and 1980s, and for the most part was restricted to selected regions in the U.S. (Beck and Rosenbaum, 1994). The drug's street name "Ecstasy" probably was coined in the early 1980s (Beck and Rosenbaum, 1994), allegedly chosen as a marketing tactic (Eisner, 1994). The degree to which the street name, Ecstasy, accurately describes the effects of MDMA has been discussed in the literature. For example, some researchers have noted that MDMA increases sexual arousal in users (Halley, Valdez, and Kaplan, n.d.; Smith, Larive, and Romanelli, 2002; Winstock, 1993) and similar claims have appeared in drug use prevention outreach material (e.g., Bad Boy Club Montreal, n.d.). Other research has suggested that MDMA users are more likely than non-MDMA users to report diminishing sexual interest (Parrott et al., 2001). However, MDMA users in that sample had used the drug frequently and also reported use of other drugs; thus, it is unclear whether the alleged effects of MDMA on sexuality are affected by consumption patterns of MMDA, other drugs, or drug interaction effects.

Some research has found that MDMA appears to increase the desire for sexual activity, although sexual performance is impaired for several users (Zemishlany, Aizenberg, and Weizman, 2001). Other accounts have supported the claim that some if not most users experience impaired sexual performance while consuming MDMA (e.g., Beck and Rosenbaum, 1994; Buffum and Moser, 1986; O'Dwyer, 1992; Saunders, 1997; Solowij, Hall, and Lee, 1992). Most of this research suggests that feelings of sensuality, openness, and collective empathy take precedence over sexual pursuits, however, Eisner (1994, p. 44) alludes to the importance of set and setting by suggesting that sexual activity during MDMA consumption is possible "when it feels appropriate to express love sexually. .

. "Buffum and Moser (1986, p. 359) found that although MDMA did not increase the desire to initiate sexual activity, the drug worked to "enhance receptivity to being sexual."

\footnotetext{
a The author acknowledges the subjectivity of the phrase "recreational drug use" 
Few studies have examined the relationship between MDMA and sexual risk taking. ${ }^{b}$ Halley, Valdez, and Kaplan (n.d.) conducted qualitative interviews with 25 MDMA users in Texas and those authors observed that most respondents had engaged in unprotected sex during MDMA episodes. Strote, Lee, and Wechsler (2002) studied drug taking (largely alcohol) and other behaviors of university students in the United States. The data were collected by mail survey and the response rate differed substantially across universities. The authors found that respondents who reported using MDMA in the past 12 months were more likely to have had multiple sex partners in the past month, compared to respondents who had not used MDMA. Those authors also found that MDMA users had higher rates of cigarette smoking and marijuana use in the past 12 months, and higher rates of binge drinking compared to respondents who had not used MDMA. Because the study was based on a cross-sectional design, causal linkages could not be ascertained. For example, Boyd, McCabe, and d'Arcy (2003) examined Ecstasy use among undergraduate students in one U.S. university. Using multivariate analysis, the authors observed that the number of sex partners significantly increased the probability of using Ecstasy. Cross-sectional data generally do not permit an assessment of whether sexual behaviors occurred during episodes of MDMA use or whether the behaviors were part of a broader lifestyle pattern that was characterized by risk taking in general.

Reports from some countries have suggested that use of MDMA as well as other drugs are factors that contribute to sexual risk taking among men who have sex with men (MSM) (Halkitis and Parsons, 2002; National Institute on Drug Abuse, 2000). Use of MDMA has been linked with unprotected sexual activity in MSM from Boston and New York City, although polydrug use was quite common among respondents (Case, 2001). Findings from some studies of MSM have suggested that use of MDMAis linked with having multiple sex partners (Klitzman et al., 2002) and unprotected anal sex (Klitzman et al., 2002; Klitzman, Pope, and Hudson, 2000; Waldo et al., 2000). Risky sexual behavior has been linked with the use of MDMA and other drugs among gay males who attend "circuit" parties (Mattison et al., 2001).

Although several MDMA users have fairly extensive drug histories, unprotected anal sex among MSM has been found to be associated with use of MDMA, controlling for use of other drugs (Klitzman, Pope, and Hudson, 2000). Still though, users of other drugs also have been found to engage in various forms of sexual risk taking. For example, a school-based self-report study of 9997 adolescents (mean = 16 years) from the Atlantic region of Canada (Poulin and Graham, 2001) found that the frequency of alcohol and cannabis use increased the likelihood of unplanned sexual intercourse. However, controlling for other variables, alcohol use (measured in terms of frequency, intoxication, binging) failed to emerge as a significant predictor for multiple sex partners or for inconsistent condom use.

A study of 100 "sexually active women" by Taylor, Fulop, and Green (1999, p. 1213) found a statistical association between the number of times intoxicated and the number of sex partners. However, cigarette smoking also was linked with the number of sexual partners; thus, the authors suggested the possibility that these patterns might have been part of a "wider cluster" of social behaviors. They did not suggest that the linkages were causal. Moreover, women in that sample reported that alcohol consumption rather than drugs increased the probability of-but did not cause-sexual activity.

Peugh and Belenko (2001) reviewed the literature on substance use, sexual arousal, and sexual function. The authors observed that most experimental and quasi-experimental studies have focused on alcohol and have relied on samples of young male adults. Still though, the authors concluded that some drugs appear to have a positive effect (perceived or otherwise) on sexual arousal, whereas other drugs reduce the likelihood of sexual arousal and impact negatively on sexual function.

\footnotetext{
b "Sexual risk taking" is also a subjective phrase. Behaviors defined as "risky" by researchers may not be perceived as such by drug users.
}

Please use the following citation: McElrath K (2005) MDMA and sexual behavior: ecstasy users' perceptions about sexuality and sexual risk. (Author postprint) in Substance Use \& Misuse, 40:9, 1461-1477, [Accessed: (date) from www.drugsandalcohol.ie] 
The purpose of the present qualitative study is two-fold. First, I describe Ecstasy users' perceptions ${ }^{c}$ about the drug's effects with respect to sensuality, sexuality, and sexual performance.

Second, I examine sexual risk taking during episodes of MDMA use.

\section{Methods}

The primary data for this study were collected through face-to-face, semi-structured interviews with current and former Ecstasy users in Northern Ireland. The criterion used for interview eligibility was any use of Ecstasy. We used this very general criterion and monitored the sample closely throughout the study, knowing that we could narrow the study criteria at a later date. ${ }^{d} \mathrm{~A}$ total of 106 respondents were interviewed, however, eight interviews were discarded because of recording or transcribing problems, leaving a sample size of 98 respondents.

Several methods were used to recruit respondents for interviews. First, announcements of the study were placed in cities, towns, and villages throughout Northern Ireland. Venues were chosen that might be described as nonthreatening and that were considered likely to be frequented by the target population, e.g., record or music shops, health centers. Second, advertisements were placed in a Northern Ireland club/music magazine. The ad was published in three monthly issues during the study period. Third, several local organizations were contacted and notified of the study. These agencies were diverse and included: youth programs, universities and student unions, sexually transmitted disease clinics, gay men and women outreach programs, young offender programs, and drug counseling or treatment centers. On several occasions, study announcements were sent to agencies by mail, and then project staff followed up with phone calls or visits in order to discuss the study in greater detail. Fourth, the interviewers recruited some respondents through their own contacts and street sources. Fifth, the researchers relied upon snowball sampling or "chain referral" techniques (Biernacki and Waldorf, 1981) whereby respondents were asked to refer friends and acquaintances. Sixth, in some instances, "recruiters" or "gatekeepers" were used and paid £10 (US $\$ 18$ ) in cash for each subsequent referral and completed interview. This method, however, was monitored closely and an upper limit of referrals (usually six) from each recruiter was established. This strategy was particularly helpful in those outlying areas where the researchers had few contacts. Clubs were not used as recruitment venues. ${ }^{e}$

Interviews were conducted between October 1997 and November 1998. Four persons, two females and two males, served as interviewers (two of whom were the Project Directors). ${ }^{\dagger}$ A research instrument was developed, piloted on the first 12 respondents, and minor revisions were made (these 12 respondents were included in the final sample). Interviews in Belfast, the largest city in Northern Ireland, generally were conducted in university offices that offered a great deal of privacy, or in other venues convenient to the respondent (e.g., private residences). Interviews with

' Because the content of Ecstasy tablets can vary considerably (DanceSafe, 2000; Sherlock et al.,
1999), perceptions concerning the effects of MDMA refer only to what users believed they have consumed.

${ }^{d}$ The vast majority of respondents who were interviewed during the first 4 months of the study resided in Belfast, the largest city in Northern Ireland. After this time we narrowed the criteria for participation in the study in an attempt to interview respondents 1) from outside Belfast or 2) former users. Current users from Belfast continued to contact us but for the most part we stopped interviewing Belfast residents for a period of approximately 2 months. At other times, we modified the study criteria so as to avoid overrepresentation of certain categories, e.g., middle-class students.

e The project directors chose deliberately to not use clubs as recruitment sources. Local folklore suggests that some paramilitary groups are closely tied to the ownership, operation, or management of various clubs. Anecdotal evidence suggests that while some paramilitary groups have been linked to drug trafficking, other paramilitary groups remain opposed to both trafficking and personal use. We believed that recruitment from clubs might have generated suspicion and may have placed potential respondents and the researchers at risk.

${ }^{\dagger} f$ All interviewers were trained during a 2-week period before the study commenced. Training topics included the nature and importance of confidentially (all staff members signed a "statement of confidentiality" and this document was framed and displayed in the main interview room), effective interviewing techniques, role playing, drug categories and effects, the local club scene (sites, locations, clientele, entrance fees, availability of alcohol, music), Ecstasy and the gay population, recruitment, and sampling issues. Additional training occurred throughout the study period during discussions in staff meetings.

Please use the following citation: McElrath K (2005) MDMA and sexual behavior: ecstasy users' perceptions about sexuality and sexual risk. (Author postprint) in Substance Use \& Misuse, 40:9, 1461-1477, [Accessed: (date) from www.drugsandalcohol.ie] 
respondents who lived outside of Belfast were conducted in private areas located within community agency sites or in private residences.

Respondents were assured of confidentiality and anonymity, and were identified by number only. Study participants were provided with a \$15 (US \$27) music or book gift certificate for a completed interview. Interviews were conducted within a 1- to 2-hour time period and focused on issues related to first, last, and usual use of Ecstasy, positive and negative drug experiences, drug use rituals and norms, health issues, and other items (McElrath and McEvoy, 1999). Respondents were encouraged to go beyond the subject area of the research instrument, when appropriate. Interviews were taped and subsequently transcribed. Demographic and drug use history data were collected at the conclusion of the in-depth interview.

\section{Description of Respondents}

Of the 98 current and former users of Ecstasy who participated in the study (Table 1), 18\% were recruited through snowball sampling, $26 \%$ were recruited by one of nine gatekeepers, $29 \%$ learned about the research through one of the study's employees, and $27 \%$ read an ad that contained information about the study. One-half of the respondents resided in Belfast at the time of the interview, 46\% lived elsewhere in Northern Ireland, and 4\% resided outside Northern Ireland. Males accounted for approximately two-thirds (69\%) of the sample, and respondents' ages ranged from 17 to 45 years $($ mean $=25$; median $=24$; mode $=23$ ). A total of $16 \%$ of the sample identified themselves as either gay or bisexual. Social class, was self-identified; $49 \%$ of respondents described themselves as working class, $46 \%$ were middle class, and $5 \%$ of respondents reported being "between" working and middle class. Just over half of the sample (57\%) were employed (either part or full time).

Table 1 Description of the sample of current and former users of $\operatorname{MDMA}(\mathrm{N}=98)$

\begin{tabular}{ll}
\hline Female & $31 \%$ \\
Gay or bisexual (self-defined) & $16 \%$ \\
Age & \\
Range & $17-45$ \\
Mean & 25 \\
Residence: & $50 \%$ \\
$\quad$ Belfast & \\
Elsewhere in N. Ireland & $46 \%$ \\
$\quad$ Outside Northern Ireland & $4 \%$ \\
Social class (self-defined) & \\
$\quad$ Working class & $49 \%$ \\
Middle class & $46 \%$ \\
$\quad$ Other & $5 \%$ \\
Employed & $57 \%$ \\
\hline
\end{tabular}

The mean age at which respondents had first used Ecstasy was 21 years (median $=20$ years mode $=19$ years; range $=13$ to 35 years). Patterns of use (e.g., weekly, monthly) fluctuated greatly over time; however, $92 \%$ of the sample had used Ecstasy during the 6-month period prior to the interview. Within that time period, $50 \%$ of the full sample had used Ecstasy less than once a month and $37 \%$ had used one to four times per month. Additionally, approximately one-half of the respondents had used Ecstasy within 2 weeks prior to the interview.

Lifetime use of Ecstasy was defined in terms of the number of separate Ecstasy experiences over the lifetime rather than the total number of tablets consumed. The term "episode" referred to a (relatively brief) time period, e.g., an evening, a weekend, during which Ecstasy was consumed. Using these definitions, our sample included a large number of respondents (i.e., 44\%) who had consumed Ecstasy on at least 100 different occasions (Table 2).

All respondents had tried cannabis ${ }^{g}$ and approximately one-third of the sample (32\%) smoked cannabis daily. The vast majority of respondents had also used LSD or magic mushrooms (90\%)

\footnotetext{
${ }^{9}$ Marijuana is not widely available in Northern Ireland. Cannabis consumed in Northern Ireland 
and amphetamines (93\%), although use of these substances was considerably lower (i.e., $30 \%$, $16 \%$, respectively) than use of Ecstasy within the 6-month period prior to the interview. Fewer respondents reported any lifetime use of cocaine/crack cocaine (45\%) or heroin (11\%). Eighty-four percent of the sample consumed beer, lager, ale, or stout on a weekly basis. Pint drinkers consumed an average of 16 pints per week (median, mode $=15$ ). Nineteen percent of the sample drank spirits and spirit drinkers consumed an average of 10 drinks per week (median, mode $=5$ ). A majority of respondents smoked at least 20 cigarettes per day in the 6 months prior to the interview.

Table 2 Drug use history

\begin{tabular}{ll}
\hline Age at onset MDMA use & \\
Range & $13-35$ \\
Mean & 21 \\
Past 6 months, MDMA use & \\
No use & $8 \%$ \\
Used <1 per month & $50 \%$ \\
Used 1-4 times per month & $37 \%$ \\
Used within 2 weeks prior to the interview & $50 \%$ \\
Used MDMA 100 times or more & $44 \%$ \\
Current users of MDMA & $77 \%$ \\
Cannabis, ever used & $100 \%$ \\
Cannabis, past 6 months & $92 \%$ \\
Cannabis, used daily in past 6 months & $32 \%$ \\
LSD/Magic mushrooms, ever used & $90 \%$ \\
LSD/Magic mushrooms, past 6 months & $30 \%$ \\
Amphetamine, ever used & $93 \%$ \\
Amphetamine, past 6 months & $16 \%$ \\
Cocaine/crack, ever used & $45 \%$ \\
Cocaine/crack, past 6 months & $7 \%$ \\
Heroin, ever used & $11 \%$ \\
Heroin, past 6 months & $2 \%$ \\
Current use of beer, lager, ale, stout & $84 \%$ \\
Weekly average & $16 \mathrm{pints}$ \\
Current use of spirits, liquor & $19 \%$ \\
Weekly average & $10 \mathrm{drinks}$ \\
Smoke $\geq 20$ cigarettes per day & $60 \%$ \\
\hline
\end{tabular}

\section{Social Settings and Use of MDMA}

Although clubs were not used to recruit respondents, nearly all respondents had frequented a club on at least one occasion when MDMA was consumed. We observed considerable variation regarding the types of clubs that respondents had frequented. For example, the social environment of some clubs appeared to cater to users of Ecstasy. Respondents reported that "techno," "hardcore," or "house" music was featured in these clubs-music characterized by rhythmic beats conducive to dancing while under the influence of MDMA. Within these clubs, respondents perceived that all or nearly all of club patrons had consumed MDMA. ${ }^{\mathrm{h}}$ Several young adult respondents, e.g., 18 to 24 years, and particularly those persons in the early stages of the Ecstasy "career," appeared to prefer this type of club.

Other clubs offered this type of music one night each week, but featured other music, e.g., "pop," and disco, at other times. Still other clubs were described in terms of pub-like atmospheres that drew club patrons whose drug preference was alcohol. These clubs were most often located in small towns and cities in Northern Ireland. Some respondents, however, traveled 1 to 2 hours in order to attend a club in Belfast. Respondents who frequented clubs Several respondents reported that additional Ecstasy tablets would be consumed at these "after-hour" parties. Persons who had

is largely hashish, which is often mixed with tobacco, rolled in a cigarette, and smoked.

\footnotetext{
${ }^{\mathrm{h}}$ We asked respondents how they could ascertain whether a person had consumed MDMA. Visible cues included large black pupils, clenched teeth, "irregular" jaw formation, extreme friendliness, particularly with strangers. often stayed until closing time and then proceeded to "house parties."
} 
been using Ecstasy for several years often preferred to consume Ecstasy in the company of close friends within their own homes or the homes of friends. Some were bored with club environments. Others grew weary of club music, preferring classical or Irish traditional music. These "house parties" were planned events whereby selected individuals within the social network would organize music, drugs, and items (e.g., comfortable clothes) needed to assist with "comedowns." Some respondents mentioned that club entrance fees, the cost of alcohol and water (often 1 or 2 pound sterling for a bottle of water-approximately 2-4 US dollars per bottle), ${ }^{i}$ and transportation costs to and from clubs contributed to their preference of consuming Ecstasy in private residences.

As stated previously, $16 \%$ of the respondents identified themselves as being gay or bisexual. However, there was no active gay "circuit" culture in Northern Ireland at the time of the study. One club venue in Belfast catered largely to gay males. A second venue, characterized by a pub-like atmosphere, drew patrons from the lesbian and female bisexual communities. A few smaller venues were located in towns outside Belfast in which clientele included several members of the gay communities. In general though, gay and bisexual respondents who lived outside Belfast reported traveling to that city for its nightlife, similar to heterosexual respondents who lived in small towns and rural settings in Northern Ireland.

\section{Findings}

\section{Feelings of Sensuality or Emotional Closeness}

A slight majority of respondents reported that Ecstasy was more associated with feelings of sensuality or deep emotions rather than sexual desire. For males in particular, these feelings defied the traditional masculine role as the seeker of sexual liaisons. A 31-year-old male described that when under the influence of Ecstasy he wished only to "hold somebody" (141). Other males enjoyed the presence of females in club settings but again had little desire to engage in sexual intercourse:

"I never thought about sex when I was on E. I used to hang out with maybe four or five girls and maybe three fellas and never even thought about it. [Ecstasy] decreased the [sexual] urge" (008, male, age 23)

"If you seen a woman in a nightclub you'd go, 'fuck she's gorgeous, she's really beautiful.' Not in a sexual way ... [it's more] emotional. You'd tell her 'you're really lovely' "(113, male, age 25)

"I can remember going to dances [where] all the women were dancing. I wanted them to go home with me, not necessarily because I wanted to have sex with them-I just wanted them around me" (302, male, age 34)

And dancing, with its associated closeness with others experiencing similar feelings, took precedence over the pursuit of sexual intercourse:

"E-l just want to go out and enjoy myself. l'd never want to go out looking for girls. I just want to dance" (025, male, age 20)

Several females also reported strong emotional feelings including sensuality, but without the desire for penetrative sex:

"You feel loved up ... I would have very little sexual encounters when I'm on E. In fact, none, because l'm more interested in the [emotional aspects]." (118, female, age 22)

\footnotetext{
' During the data collection phase, a number of respondents reported that water taps or faucets were turned off in the public restrooms within certain clubs. There were also reports of the availability of hot water only in public restrooms. In several clubs, free glasses of water were not available from bar staff; rather, only bottled water was available, and sold for profit.
} 
"There's sexual energy about the whole thing but it's not about finding someone ... You want to be close to people and there's a touchy feely thing about it but it's not about penetrating sex" (007, female, age 30$)$

These findings are consistent with data reported in other cultures (e.g., Beck and Rosenbaum, 1994), that is, several Ecstasy users report feelings of emotional and perhaps physical closeness associated with sensuality while consuming the drug.

\section{Sexual Arousal}

Although several respondents did not experience feelings of sexual arousal during Ecstasy episodes, other respondents, males in particular, reported having engaged in sexual intercourse while consuming Ecstasy. Others attempted to engage in sexual activity but reported their inability to achieve erection while consuming Ecstasy. This finding is consistent with previous research (e.g., Buffum and Moser, 1986). Difficulties in achieving erection or being unable to ejaculate appeared to affect younger males (e.g., aged 25 and younger) more often than older males (e.g., 26 to 38 years). For these young males, it was not as if MDMA-related feelings of sensuality took precedence over feelings of sexuality. Rather, many who reported these difficulties had attempted to engage in sexual intercourse during $E$ episodes but found it physically impossible to do so:

“I just couldn't get an erection . . . for six hours" (122, male, aged 25)

"Sometimes it's not even 'til the next morning before you can even have sex" (127, male, age 23)

"You're lucky if you can even get it up when you're on E" (144, age 20)

Among some male and female respondents, however, sexual desire was enhanced "big style" (022, female, age 23; 215, male, age 20) when consuming Ecstasy, whereby one could be "at it the whole night" (216, male, age 18):

"I think that sex is brilliant on Es. You want to shag [i.e., have sexual intercourse] for Ireland all night, and you don't care ... Without wanting to be crude but it definitely would make me feel horny, and feel that I want sex" (018, female, age 30)

Interviewer: "Have you ever used it specifically for sex?"

"Yes, l've done it a few times. Sometimes we would have, maybe had friends around for dinner, or we would have had dinner ourselves . . . a bottle of wine, [and] used it [MDMA] for the sexual side of things."

Males who had experienced sexual intercourse while consuming MDMA appeared to distinguish between two kinds of sexual activity. MDMA-related sex was unusual or different from other forms of sex. For example, a male respondent described MDMA-related sexual desire with a girlfriend:

"It wasn't like the horn you get with speed, it was a lovey-lovey kind of thing" (139, male, age 28)

Respondents suggested that (similar to other drugs) Ecstasy decreased inhibitions, enhanced emotions, and boosted levels of confidence $(021,028,031)$. The combination of these factors as well as the setting in which Ecstasy use occurs, appeared to contribute to the likelihood that penetrative sex might occur:

"[Ecstasy] made it easier [to interact with friends and strangers].You felt sexual, it heightened your sexuality. Sex was very good on E" (010, male, age 27) 
These sexual encounters tended to take place in private residences, either after people had left clubs or during planned house parties:

"You get very horny on E." (024, male age 24)

Interviewer: "Do you act on it?"

"[In the clubs] there's no slow music so you don't act on it. You might after a disco like,

at a house party, but that's about it—not in the middle of a disco."

Research conducted elsewhere has found that some MDMA users reported being more open to exploring new forms of sexuality (Beck and Rosenbaum, 1994; Buffum and Moser, 1986). Similar results were observed in the present study. For example, a few respondents reported using Ecstasy as a "sexual aid" with a steady partner (018, female, age 30). A male respondent reported that:

"If I went to a club dancing, it [MDMA] would be used for energy, the rush and the whole sort of ecstatic feeling of it. But l've taken $E$ quite a few times and not danced.

With my ex-girlfriend we would take $E$ and just enjoy ourselves. We'd go out for a walk, go to the park or go to bed." (138).

Interviewer: "Tell me about that. Would it affect sex in any way?"

"Definitely. I thought it heightened all the sensations of it. It seems to make it a lot more crystal clear."

Interviewer: "Because some people have told us that have had problems with E ..."

"Getting erections?"

Interviewer: "Yeah."

"Haven't really had problems with that."

Interviewer: "So generally do you think it is a good sexual aid?"

"Yeah definitely."

Individuals mentioned feeling more open-minded about sexual exploration during Ecstasy episodes (204, male, age 27). A 28-year old male respondent described that Ecstasy heightened sexual feelings for him and that:

"You want to creatively indulge yourself in new forms of sex" (032).

Some female respondents reported that new forms of sexuality included bisexuality. For example, a 29-year old lesbian reported that groups of Ecstasy users often experienced intensive relaxation in an "accepting environment" so that exploring bisexuality is "easier . . . in an E-ed up environment" (019). A 23-year-old bisexual female recalled that the last 10 women with whom she had engaged sexually, were straight women who had been using Ecstasy or some other illicit drug. She reported that she had used Ecstasy specifically for sexual purposes:

"I've been sitting in the house at night, and said, 'let's do some Es.' Lying there taking $E$ and doing the whole 'go to bed thing,' listening to music, dance music like. You've about 5 CDs on continuous play. You go to bed about 10:00 at night and that's it 'til about 7 or 8:00 the next morning. Then you spend the whole of the next day recovering in bed" (022).

Interviewer: "And do you feel it enhances sexual feelings?"

"Oh, yeah. Anything that you normally wouldn't even think about-much more perverted-kinky to a certain extent. You can break down the barriers and for once have completely uninhibited sex. I do believe it has to be done by any gay girl and it's brilliant for relieving tensions and stress."

A heterosexual female reported similar feelings:

"People seem to be an awful lot more attractive [when I am using Ecstasy] . . . It makes you feel a lot more open to suggestions ... you tend to go with things a lot easier." (029) 
In sum, the majority of male and female respondents reported that Ecstasy increased feelings of sensuality and produced strong feelings of empathy and emotional closeness, but often without the need or desire for or culmination of sexual activity. Alternatively, a second and apparently distinct group of users reported that Ecstasy increased sexual desire and contributed to sexual fulfilment. These diverse feelings may simply reflect general differences in sexual interest. That is, the degree of sexual interest can vary among individuals regardless of whether drugs such as Ecstasy are consumed. For example, respondents explained that their sexual feelings (or lack of) when consuming Ecstasy often differed from the feelings of their friends or acquaintances (009, male, age 21). A 30-year-old male reported:

“There's another mate of mine that has said, 'I'm not taking E again, I can't get a hard on,' whereas I could rap the door with it" (205)

"Most people say it does make them more sexual, but in my case I didn't find that" (131, male, age 29)

A 30-year-old female respondent who experienced sexual desire while consuming Ecstasy recalled the differences in MDMA-related sexual interest among friends:

"[He wasn't] interested in sex at all ... he would just sit all night and play music. I was like, 'How does he do that'? "(018)

Although several respondents had consumed alcohol—sometimes in large amounts—during Ecstasy episodes, others avoided alcohol entirely. Heavy drinkers were just as likely as light drinkers or non-drinkers, to engage in or attempt to engage in sexual activity. A large percentage of the sample-about $44 \%$ - had consumed MDMA on at least 100 different occasions. Similar to the apparent non-effect of alcohol, the data suggest that feelings of sensuality and sexuality did not differ between persons who had consumed MDMA at least 100 times and persons who had consumed the drug a few times.

\section{Sexual Risk Behaviors}

Several of those respondents who had engaged in sexual activity while consuming Ecstasy reported having engaged in behaviors that pose risk for infectious disease. These sexual risk takers were more often younger, e.g., 18 to 25 years old, and this finding is consistent with research that shows that younger IDUs engage in considerably more risk behaviors for infectious disease than older IDUs (Fuller et al., 2003). Some respondents reported having multiple sex partners whereby the choice to do so was perceived to be influenced by the effects of Ecstasy:

"I'm terrible . . like I will just hop on anything when I'm on E" (023, female, age 21)

Some respondents reported having engaged in "one night stands" with strangers or with distant acquaintances during Ecstasy episodes:

"She was the same. She'd taken it and she wouldn't normally done it with me, so it was just one of those things ... it wasn't a big deal . . . there are no kids on the way so we were OK" (108, male, age 18)

A number of male and female respondents voiced next-day regrets about their sexual activity during MDMA episodes (014, male, age 25):

"I've woke up beside people thinking, 'What the fuck did I do last night?' People's barriers are down." (022, female, age 23)

"You just feel really loved up and it's like, some bloke, and then the next morning you're thinking, 'Oh no"' (105, female, age 20) 
Several respondents reported that some or many of their friends engaged in unsafe sex while during E episodes (136, male, age 21; 123, male, age 17). Again, these respondents tended to be younger and it is likely that their friends were in the same age group. A 24-year old self-defined lesbian recalled an earlier living situation:

"I lived in a house with six heterosexual girls and a gay man . . . they all did have unsafe sex with people we had copped off with [met and left with] in a club" (125).

Many respondents who engaged in sexual activity during Ecstasy episodes did so during the latter stages of the evening :

Interviewer: "How do you come down after taking E?"

"Usually it's according to whether l've copped off. If I have, sex is quite a good way of dealing with a comedown. If not, l'll maybe go to a party and chill-out, skin up [roll a joint], do the talking thing to everybody, and then go home, listen to music and fall asleep" (022, female, age 23).

Condom use was rare during Ecstasy episodes that involved sexual intercourse; however, few respondents reported using condoms during sexual encounters when drugs had not been consumed. Some respondents reported using condoms with strangers but not with partners (215, male, age 20).

Because a number of respondents tended to consume alcohol as well as other substances during MDMA episodes, it is difficult to disentangle the effects of alcohol and MDMA on sexual risk behaviors. We asked respondents their views on the influence of MDMA compared to alcohol in relation to sexual risk taking. Respondents' views were mixed. Some claimed that sexual risk taking occurred more often while consuming Ecstasy compared to alcohol:

"You are full of the horn, your emotions are running, more [so] than with alcohol" (115, male, age 29, used Ecstasy on 100 occasions or more)

A male respondent reported that use of Ecstasy increased the likelihood of unprotected sex, not because of increased sexual desire but because "you want to be a romantic fuck" (212, age 19, used Ecstasy 100 times or more). Others reported that use of alcohol was far more likely than use of Ecstasy to contribute to sexual risk taking (014, male, age 25, used Ecstasy 100 times or more):

The way that l've taken $\mathrm{E}$, remembering everything and being in control . . . I think [sexual risk taking] would be more so with alcohol" (124, female, age 21, used Ecstasy on four occasions)

"I'd say you have more control on $E$ than if were pissed [intoxicated]. I think you're much more conscious of what you're doing" (007, female, age 30, used Ecstasy on 100 occasions or more)

\section{Discussion}

\section{The Role of MDMA, Other Drugs, and Sexual Behavior}

The findings suggest that the perceived relationship between MDMA and sexual behaviour differed among respondents. Many respondents reported that although MDMA increased feelings of sensuality and intimacy, the pursuit of sexual relationships was minimal. Indeed, some respondents suggested that MDMA reduced the likelihood of sexual performance. Other respondents perceived that MDMA contributed to a greater likelihood of engaging in sexual activity and some reported that the drug added to sexual enjoyment. Lifetime consumption of MDMA and the amount of alcohol consumed during Ecstasy episodes did not appear to affect whether respondents reported feelings of sexuality vs. sensuality.

In their review of the literature of substance use and sexual function, Peugh and Belenko (2001) concluded that substances can affect both sexual arousal and sexual functioning. However, the 
authors found that the effects appear to depend on the particular substance ingested, the dosage consumed, as well as individual perceptions or expectations of drug effects. Other research has found that several users of MDMA consume other substances during the same drug "episode" (Sherlock and Conner, 1999; Shewan, Dalgarno, and Reith, 2000; Topp, Barker, and Degenhardt, 2004). In the present study, several persons regularly consumed alcohol during Ecstasy episodes and a number of respondents reported using cannabis shortly before MDMA was consumed during the Ecstasy episode or to assist with "coming down" from Ecstasy. Although the findings suggest that alcohol consumption patterns appeared to have little effect on sexual behavior, the interaction effects between or among MDMA, alcohol, and cannabis could not be examined.

To the author's knowledge, research that examines the interaction effects of these substances on sexual behavior has not been undertaken. Peugh and Belenko (2001) found that "heavy" consumption of alcohol tends to reduce sexual arousal, particularly among men. However, those authors also reviewed evidence that suggested that marijuana can increase sexual arousal. Given these diverse effects on sexual arousal, what is the outcome when cannabis and alcohol are combined? And does MDMA combined with these other substances produce a different outcome altogether? The answers lie with future research. Prospective data might be needed to explore these issues in greater detail because recall might be affected through retrospective accounts of events that involve heavy consumption of multiple substances. Moreover, detailed information on the timing of drug and alcohol consumption patterns and sexual activity are needed in order to better understand the relationship.

\section{MDMA As a Sexual "Aid"}

A number of respondents in the present study reported that MDMA enhanced sexual relations by reducing sexual inhibitions or boosting sexual confidence. Some users reported that they had used MDMA as a sexual aid. A few claimed that the drug encouraged them to explore new forms of sexuality, similar to research findings reported from a Texas study (Halley, Valdez, and Kaplan, n.d.). Of particular interest is that gay and bisexual women appeared to be more likely than other respondents to use MDMA for sexual enhancement, although the small number of gay and bisexual women in the sample prohibits accurate conclusions from being drawn. A study of "club drug" use among Chicago adults found that gay and bisexual women were more likely to use "club drugs" (e.g., MDMA) compared to heterosexual women (Fendrich et al., 2003). Those authors found that the use of "club drugs" did not differ significantly between gay and heterosexual males. Taken together, these findings suggest the need for further research into the linkages between MDMA and sexual behavior of gay and bisexual women.

\section{MDMA and Sexual Risk}

We asked respondents about risk behaviors regarding sexual activity. It should be noted that several respondents required clarity with regards to this question, suggesting that behaviors that we view as risky may not be perceived as such among drug users. Clearly the concept of "risk" is subjective. Nevertheless, our perception of sexual risk suggests that although some respondents tended to avoid these behaviors, others engaged in diverse sexual risk taking during MDMA episodes, e.g., having multiple sex partners, choosing sex partners who were acquaintances or strangers. These findings are consistent with earlier research that found that frequent sexual activity with multiple partners was common in the Dallas club scene during the 1980s (Beck and Rosenbaum, 1994). Although gay and lesbian women appeared to engage more with multiple sex partners, at least one respondent reported that this type of behavior was far more risky for heterosexuals and gay males than for lesbians. She perceived that her sexual behaviors posed little risk for the transmission of infectious disease.

\section{Prevention and Harm Reduction}

In one sense, sexual risk behaviors fit neatly within the collective circle of Ecstasy users. For example, failing to use a condom denotes trust of another and condom usage is inconsistent with the "loved up" trusting environment in which Ecstasy is often consumed. In fact, a male respondent reported that he used condoms during sexual encounters when Ecstasy had not been consumed, but that he failed to do so during Ecstasy episodes because "you just have this total love buzz" (210, age 22, used Ecstasy 100 times or more). Having multiple sex partners within a specified 
period of time reinforces the collective bond that characterizes many subcultures of which Ecstasy users are members. Condom usage among respondents was rare and unprotected sexual intercourse increases the likelihood of infectious disease. As part of their "healthy settings" approach, Bellis, Hughes, and Lowey (2002) suggested that condoms should be available in club settings. At the time of the study, condom machines were located in restrooms of several clubs and pubs. However, the machines were often placed in semi-public areas of restrooms where privacy can be compromised. Condom machines are a "passive" harm reduction tool. Alternatively, outreach work within clubs represents a proactive method for promoting good sexual health within clubs. Club owners and managers should assume more responsibility for promoting good sexual health by employing or providing financial contributions to employ outreach workers within club settings. This study found, however, that some users prefer to consume Ecstasy in other places, e.g., private residences. This group might be best reached through peer education.

\section{Limitations of the Study and Suggestions for Future Research}

The data for this study were collected from current and former Ecstasy users between October 1997 and November 1998. It is likely that the scene has changed since that time. We know, for example, that Ecstasy tablets sold for approximately $£ 10$ (approximately 18 US dollars) each during the data collection stage. Anecdotal reports now suggest that they sell for $£ 5$ each. Researchers in England have noted that the use of alcohol in club settings has become more common in recent years (Measham, Aldridge, and Parker, 2001), whereas in the early 1990s, alcohol in club settingswas used less frequently and for some individuals, was not consumed at all (Henderson, 2000). It is possible that similar changes have occurred in Northern Ireland. Research that investigates linkages between sexual behavior and drug taking generally has not been undertaken in Northern Ireland. It is therefore unclear whether the findings observed in this study would apply currently.

Among the several respondents who initiated Ecstasy use in Northern Ireland, none had done so before 1991. Several respondents reported that the "scene" surfaced in Northern Ireland in the early 1990s; thus by the time the study commenced the Ecstasy scene (or scenes) was firmly entrenched in various (primarily) youth subcultures. In comparison to several European countries including those regions in close geographic proximity to Northern Ireland, e.g., the south of Ireland, England, and Scotland, substantial increases in the use of MDMA in the United States were not observed until the late 1990s (Agar, Schacht, and Reisinger, 2003). The current Ecstasy "scene" in the United States has an approximate time span that is similar to the Northern Ireland scene observed when this study was undertaken. Future qualitative research might focus on sexual behaviors of MDMA users in the United States so that comparisons can be drawn. With few exceptions (see for example, the work by Carafat et al., 1998), cross-cultural studies of Ecstasy use have not been undertaken. Such studies would add to our understanding of the degree to which wider social and political contexts can influence patterns of MDMA use and sexual behaviors.

\section{THE AUTHOR}

Karen McElrath Ph.D., is a Reader in the School of Sociology and Social Policy, Queen's University, Belfast. Her current research focuses on stigma and risk among injecting drug users, drug use and political conflict, and measurement issues. She is the co-editor (with James Inciardi) of The American Drug Scene (Roxbury, 4th edition) and editor of HIV and AIDS: A Global View (Greenwood).

\section{References}

Agar, M., Schacht Reisinger, H. (2003). Going for the global: The case of Ecstasy. Human Organization 62:1-11.

Bad Boy Club Montreal (n.d.). Recreational Drug Use and the Circuit Parties. Available at http://members.aol.com/tjfronczak/drugs,htm. 
Beck, J., Rosenbaum, M. (1994). The Pursuit of Ecstasy: The MDMA Experience. Albany: State University of New York Press.

Bellis, M. A., Hughes, K., Lowey, H. (2002). Healthy nightclubs and recreational substance use: from a harm minimisation to a healthy settings approach. Addictive Behaviors 27:1025-1035.

Biernacki, P., Waldorf, D. (1981). Snowball sampling: Problems and techniques of chain referral sampling. Sociological Methods and Research 10:141-163.

Boyd, C. J., McCabe, S. E., d'Arcy, H. (2003). Ecstasy use among undergraduates: Gender, race and sexual identity. Journal of Substance Abuse Treatment 24:209-215.

Buffum, J., Moser, C. (1986). MDMA and human sexual function. Journal of Psychoactive Drugs 18:355-359.

Carafat, A., Stocco, P., Mendes, F., Simon, J., van de Wijngaart, G., Pau Sureda, M., Palmer, A., Maalst'e, N., Zavatti, P. (1998). Characteristics and Social Representation of Ecstasy in Europe. Palma de Mallorca (Spain): IREFREA and European Commission.

Case, P. (2001). Patterns of MDMA use among men who have sex with men in Boston and New York City. MDMA/Ecstasy Research: Advances, Challenges, Future Directions, Bethesda, MD, July 19-20.

DanceSafe. (2000). May-July 2000 results. http://www.dancesafe.org/past lab results/results mayjuly2000.htm

Eisner, B. (1994). Ecstasy: The MDMA Story. 2nd Ed. Berkeley: Ronin.

Fendrich, M., Wislar, J. S., Johnson, T. P., Hubbell, A. (2003). A contextual profile of club drug use among adults in Chicago. Addiction 98:1693-1703.

Fuller, C. M., Vlahov, D., Latkin, C. A., Ompad, D. C., Celentano, D. D., Strathdee, S. A. (2003). Social circumstances of initiation of injection drug use and early shooting gallery attendance: Implication for HIV intervention among adolescent and young adult injection drug users. Journal of Acquired Immune Deficiency Syndromes 32:86-93.

Halkitis, P. N., Parsons, J. T. (2002). Recreational drug use and HIV-risk sexual behavior among men frequenting gay social venues. Journal of Gay and Lesbian Social Services 14:19-38.

Halley, J. A., Valdez, A., Kaplan, C. D. (n.d.) Ecstasy use and sexual behavior in the south Texas club scene: A study of Mexican American Ecstasy users. Available at: http://www.drugtext.org/library/articles.halley.htm

Henderson, S. (2000). Drugs and culture: The question of gender. In: South, N., ed. Drugs: Cultures, Controls and Everyday Life. London: Sage, pp. 36-48.

Klitzman, R. L., Greenberg, J. D., Pollack, L. M., Dolezal, C. (2002). MDMA ('ecstasy') use, and its association with high risk behaviors, mental health, and other factors among gay/bisexual men in New York City. Drug and Alcohol Dependence 66:115-125.

Klitzman, R. L., Pope, H. G., Hudson, J. I. (2000). MDMA ("Ecstasy") abuse and high-risk sexual behaviors among 169 gay and bisexual men. American Journal of Psychiatry 157:1162-1164.

Mattison, A., Ross, M.W.,Wolfson, T., Franklin, D., HNRC Group. (2001). Circuit party attendance, club drug use, and unsafe sex in gay men. Journal of Substance Abuse 13:119-126.

McElrath, K., McEvoy, K. (1999). Ecstasy Use in Northern Ireland. Belfast: Stationery Office. Measham, F., Aldridge, J., Parker, H. (2001). Dancing on Drugs: Risk, Health and Hedonism in the British Club Scene. London: Free Association.

National Institute on Drug Abuse. (2000). United States-Eastern Europe regional meeting on Methamphetamine and ecstasy use. Visegr'ad, Hungary, March 31 to April 2.

O'Dwyer, T. (1992). The phenomenology of ecstasy use. St. Thomas' Hospital. Morpeth/Leeds Addiction Unit. In: Saunders, N., ed. $E$ is for Ecstasy. Appendix 1.Available at: http://www.ecstasy.org/books/e4x/e4x.ap.01/e4x.ap.01.037.html 
Parrott, A. C., Milani, R. M., Parmar, R., Turner, J. D. (2001). Recreational ecstasy/MDMA and other drug users from the UK and Italy: Psychiatric symptoms and psychobiological problems. Psychopharmacology 159:77-82.

Peugh, J., Belenko, S. (2001). Alcohol, drugs and sexual function: A review. Journal of Psychoactive Drugs 33:223-232.

Poulin, C., Graham, L. (2001). The association between substance use, unplanned sexual intercourse and other sexual behaviours among adolescent students. Addiction 96:607-621.

Saunders, N. (1997). Ecstasy Reconsidered. London: Author.

Sherlock, K., Conner,M. (1999). Patterns of ecstasy use amongst club-goers on the UK 'dance scene.' International Journal of Drug Policy 10:117-129.

Sherlock, K., Wolff, K., Hay, A. W. M., Conner, M. (1999). Analysis of illicit ecstasy tablets: Implications for clinical management in the accident and emergency department. Journal of Accident and Emergency Medicine 16:194-197.

Shewan, D., Dalgarno, P., Reith, G. (2000). Perceived risk and risk reduction among ecstasy users: The role of drug, set and setting. International Journal of Drug Policy 10:431-453.

Shulgin, A. T. (1986). The background and chemistry of MDMA. Journal of Psychoactive Drugs 18:291-304.

Smith, K. M., Larive, L. L., Romanelli, F. (2002). Club drugs: Methylenedioxymethamphetamine, flunitrazepam, Ketamine hydrochloride, and gamma-hydroxybutyrate. American Journal of Health

Systems Pharmacy 59:1067-1076.

Solowij, N., Hall,W., Lee, N. (1992). Recreational MDMA use in Sydney: A profile of ecstasy users and their experiences with the drug. British Journal of Addiction 87:1161-1172.

Strote, J., Lee, J. E.,Wechsler, H. (2002). Increasing MDMA use among college students: Results of a national survey. Journal of Adolescent Health 30:64-72.

Taylor, J., Fulop, N., Green, J. (1999). Drink, illicit drugs and unsafe sex in women. Addiction 94:1209-1218.

Topp, L., Barker, B., Degenhardt, L. (2004). The external validity of results derived from ecstasy users recruited using purposive sampling strategies. Drug and Alcohol Dependence 73:33-40.

Waldo, C. R., McFarland,W., Katz, M. H., MacKellar, D., Valleroy, L. A. (2000). Very young gay and bisexual men are at risk for HIV infection: The San Francisco Bay Area Young Men's Survey II. Journal of Acquired Immune Deficiency Syndrome 24:168-174.

Winstock, A. (1993). Unpublished data, Hammersmith Hospital, Royal Postgraduate Medical School. Cited in Saunders, N., Is E ed. for Ecstasy. Appendix. Available at: http://www.ecstasy.org/books/e4x/e4x.ap.01/e4x.ap.01.045.html

Zemishlany, Z., Aizenberg, D., Weizman, A. (2001). Subjective effects of MDMA ("ecstasy") on human sexual function. European Psychiatry 16:127-130. 